\title{
Tecnología presente, padres ausentes
}

\section{This technology, absent fathers}

Eve Deyanira Reyna Legarreta ${ }^{1}$

Diana Laura Betancourt Ochoa ${ }^{2}$

Paola Elizabeth Rojas Torres ${ }^{3}$

\section{Resumen}

La importancia de las relaciones interpersonales de los niños en su etapa de preescolar principalmente, toma un papel con una relevancia enorme, debido a que en estas edades es donde se forma la personalidad con características, habilidades y cualidades específicas, que diferentes situaciones amoldan y atribuyen a esta, hasta consolidarse y no poder modificarse de una manera fácil. Muchos estímulos externos forman la personalidad, siendo uno de ellos la tecnología, que en la actualidad es uno de los mayores factores que repercuten en la vida de cualquier persona. Lo trascendente aquí es el mal uso que se le da y que cada vez es más común en personas de corta edad y sin una supervisión debida, el problema lo encontramos cuando nos damos cuenta de las repercusiones que tiene, como lo es el aislamiento, relaciones interpersonales débiles e inestables, problemas de salud físicos, sedentarismo, indisciplina, por otro lado existe el riesgo de convertirse en una adicción en casos extremos. Por esta razón es importante la intervención por parte de docentes y padres de familia para poder formar personas que sean conscientes del uso de la tecnología como una herramienta. Del mismo

1 Eve Deyanira Reyna Legarreta. Institución Benemérita y Centenaria Escuela Normal del Estado de Chihuahua Profesor Luis Urías Belderráin, México. Licenciatura en educación preescolar. Correo electrónico: evereyna7@gmail.com ID: http://orcid.org/0000-0001-7896-9194

2 Diana Laura Betancourt Ochoa. Institución Benemérita y Centenaria Escuela Normal del Estado de Chihuahua Profesor Luis Urías Belderráin, México. Licenciatura en educación preescolar. Correo electrónico: betancourtdiana.96@gmail.com

ID: http://orcid.org/0000-0001-6022-9999

3 Paola Elizabeth Rojas Torres. Institución Benemérita y Centenaria Escuela Normal del Estado de Chihuahua Profesor Luis Urías Belderráin, México. Licenciatura en educación preescolar. Correo electrónico: paolaerojast@gmail.com

ID: http://orcid.org/0000-0002-1533-4252 
RECIE. Revista Electrónica Científica de Investigación Educativa Vol. 4, núm. 2, enero-diciembre 2019, pp. 1201-1208.

modo que identifiquen la importancia de las relaciones socio-afectivas y el lado humano que nos caracteriza a todos como parte de nuestra naturaleza y desarrollo.

\title{
Palabras clave
}

Tecnología, relaciones interpersonales, familia, afectividad, infancia.

\begin{abstract}
The importance of interpersonal relationships of children at the preschool stage mainly takes a role with a relevance that is huge, since in these ages is where the personality is formed with characteristics, skills and specific qualities, which different situations adapted and attributed to this, to consolidate and not be able to modify in an easy way. Many external stimuli are personality, one of them being the technology, which currently is one of the major factors affecting the life of any person. The transcendent here is the misuse that is given and that increasingly is more common in young people and without proper supervision, we find the problem when we realize the implications, such as insulation, weak and unstable interpersonal relationships, physical health, sedentary lifestyle, indiscipline, on the other hand there is the risk of becoming an addiction in extreme cases. For this reason, intervention by teachers and parents is important to be able to form people who are aware of the use of technology as a tool. In the same way that identifies the importance of the socio-affective relationships and the human side that characterizes us all as part of our nature and development.
\end{abstract}

\section{Keywords}

Technology, interpersonal relationships, family, affectivity, childhood.

\section{Introducción}

Con el propósito de ayudar a la comunidad escolar durante un año, se planteó un proyecto de intervención socio-educativa el cual fue centrado en una problemática activa dentro de este entorno, gestionando y planificando estrategias de acción para su logro. El proceso de trabajo para dicho proyecto debe de componerse de una serie de pasos que sirvan como antecedente al trabajo que después se complementará con los resultados finales, sin este proceso el trabajo quedaría incompleto y sería irrelevante pues las ideas y resultados estarían inconclusos.

De acuerdo a las observaciones y los instrumentos de diagnóstico aplicados a la población escolar, siendo estos las entrevistas aplicadas de 
manera indirecta a los padres de familia y al personal docente del Jardín, permitió conocer rasgos que dieran un panorama amplio de lo que se vive en casa. Por otro lado, a los alumnos se les aplicaron diferentes estrategias y entrevistas de manera directa sobre el tema controversial que fue "la familia" y "la tecnología" para que de igual manera expresaran y describieran situaciones que se viven en su hogar y entorno familiar acerca de la convivencia y el uso de las TIC.

Por tanto, se determinó la problemática a tratar y en base a esto se tuvo un periodo de trabajo en el cual se plantearon los objetivos, metas y la manera en que se trataría de dar solución y crear conciencia en ambas partes (padres e hijos), que a continuación, se mostrarán a detalle, así como los resultados y el transcurso de dicho proyecto, con el fin de compartir la experiencia vivida y los resultados obtenidos de esta.

\section{Importancia de las emociones en edad preescolar}

Durante la infancia las emociones juegan un papel fundamental para el desarrollo adecuado del niño. Desde el nacimiento el infante tiene la oportunidad de desarrollarse socialmente siempre y cuando haya alguien que lo guíe. "La necesidad de ser sostenido emocionalmente por otro y la búsqueda e interés en la relación humana son rasgos de salud mental que el niño manifiesta desde el comienzo de su vida." (Bowlby, J., 1989: Una base segura)

Dentro del Jardín de Niños ubicado en la zona centro de la ciudad de Chihuahua, se implementó un proyecto que parte de la siguiente problemática: “¿Cómo la tecnología ha repercutido negativamente en las relaciones socio afectivas entre padres e hijos?". Las representantes de dicho proyecto decidieron actuar ante esto por la poca relación que se manifestaba entre dichos actores, y a causa de esa escasez de comunicación y relación los infantes mostraron efectos colaterales que perjudican su comportamiento, desempeño y hasta su confianza en las actividades diarias en la institución. Esta situación se detectó de acuerdo a los resultados que los instrumentos de diagnóstico arrojaron, como lo fueron: entrevistas directas e indirectas, observaciones diarias realizadas desde la primera jornada de práctica y mediante la convivencia diaria con los alumnos y padres de familia al realizar comentarios inconscientes sobre esto.

Henao, G. Ramírez, C. y Ramírez, L. (2007) menciona que la importancia de la familia en la socialización y desarrollo durante los niños de 1 a 6 años, muestran cómo la combinación de costumbres y hábitos de crianza de los padres y madres. La sensibilidad que tienen hacia las necesidades del infante, la aceptación de su individualidad, el afecto que se expresa y los 
RECIE. Revista Electrónica Científica de Investigación Educativa Vol. 4, núm. 2, enero-diciembre 2019, pp. 1201-1208.

mecanismos de control son base fundamental para regular el comportamiento de los mismos esto ocasiona que se logre un equilibrio emocional.

Las emociones se desarrollan desde la infancia, es la etapa en donde más inestables se encuentran por ende el buen o mal desarrollo de estas causan impacto a lo largo de su vida, creando la personalidad y la autoestima, reflejándose en su seguridad y desenvolvimiento ante las situaciones que la vida les presenta.

\section{Vínculo afectivo entre padres e hijos}

El lugar en donde los infantes pueden recibir un afecto significativo es en casa, en donde los padres son los principales actores que pueden y deben proporcionar esto a sus hijos. Crear un vínculo afectivo con ellos es algo sumamente importante, porque construye un ambiente de confianza, seguridad y estabilidad en el hogar, que como resultado trae consigo un sinfín de beneficios tanto para el niño como para la familia. "El afecto es una necesidad, quizás, la más importante para el desarrollo emocional de los niños, puesto que deriva una buena autoestima, seguridad en sí mismo(a), manejo adecuado de las relaciones interpersonales, entre otros aspectos" (Manfred Max-Neef, 1996)

Dar tiempo a los hijos y escucharlos, es dar importancia a lo que sienten, a lo que experimentan y piensan, que lleva a conocerlos mejor. Decirles lo importantes que son y dar afecto, les genera bienestar, no solo a ellos sino a los padres también. Tener esta estabilidad en ellos mismos y con los demás, los lleva a desenvolverse mejor en cualquier ámbito y superar retos de cualquier origen. Como menciona Judith Izquierdo (2013) que la construcción de un vínculo adecuado implica expresar abiertamente el afecto que sentimos, hacer sentir a la persona querida y aceptada tanto por las cualidades que nos gustan de ella, como por las que no. Conlleva crear espacios de intimidad, dedicar tiempo e implicarnos activamente en conseguir el bienestar del otro, escucharnos, abrazarnos.

Por esta razón el proyecto giró en torno a los padres de familia, con el fin de concientizarlos sobre la importancia de la relación afectiva entre ellos y sus hijos y que conocieran cómo el uso inadecuado de las redes sociales y la tecnología la están afectando directamente, ya que en la actualidad están al alcance de cualquiera y ya no se miran como un lujo sino como una necesidad, pero una necesidad a la que se le debe de dar un buen uso.

Inmerso en lo anterior se esperó que los padres de familia conocieran la importancia de mantener esta relación afectiva con sus hijos, mediante 
actividades de impacto directo. Por otro lado, se pretendió que los alumnos externaran sus sentimientos ante diferentes situaciones de la vida diaria y dentro de su entorno familiar y darlas a conocer a los padres de familia para así generar un ambiente de confianza entre ellos.

\section{Era de las redes sociales}

Las redes sociales son los protagonistas y encargados de que los usuarios y las comunidades que la conforman estén en plena comunicación, para el intercambio de información. Actualmente, las redes sociales son los nuevos espacios en los que nos relacionamos y en los que construimos nuestra propia identidad" (Orihuela, 2008, p.59).

Las docentes en formación, tienen una tarea valiosa con las generaciones del futuro, pues son las encargadas de generar en ellas conocimientos y desarrollar habilidades que favorezcan en ellos características propias que los diferencien, los unos con los otros. Esta tarea que tienen en sus manos es de suma importancia pues son instrumentos de guía para los alumnos, los más pequeños miembros de la sociedad.

En la actualidad es fácil tener acceso a un aparato tecnológico y por ende a una red social, que está de más decir que en ésta época, el no estar dentro de una significa no pertenecer, en cierto modo a la sociedad, pues lamentablemente se están dañando las relaciones interpersonales.

Este proyecto se hizo con el fin de generar conciencia y tocar puntos clave para abrir espacios en los cuales se diera el diálogo y la convivencia sana y afectiva entre las familias del entorno en el que estábamos sumergidas, pues como ya es de conocimiento público, las redes sociales están abriendo puertas a la comunicación internauta, pero la interpersonal se está perdiendo, la cual es importante pues no solo favorece a las buenas relaciones, sino que también a un desarrollo propio

\section{Impacto de la tecnología dentro de la familia}

Si bien se sabe, el hogar es la primera escuela, en donde los infantes absorben la mayor parte del tiempo los conocimientos, costumbres y tradiciones que arraigan de sus familias. Como se mencionó anteriormente, la era de las redes sociales y la tecnología ha invadido de manera revolucionaria el mundo en el que los niños se encuentran inmersos y con ello repercutiendo directamente en las relaciones socio afectivas entre el entorno familiar donde se encuentran. "La familia como usuario de esta tecnología se convierte en un agente activo en el posicionamiento del mismo en la cultura, internet a su vez en esta relación interviene en el desarrollo de la vida familiar, modificando 
hábitos y costumbres de los miembros que la componen" (Winocur, 2009; Tully, 2007; Kurzweil, 2007 citado por Barrera \& Duque, 2014, p. 31).

Partiendo del diagnóstico, se decidió atender de manera puntual los rasgos más fuertes de la problemática que arrojó la investigación que se centra en "La verdadera importancia y el impacto de las relaciones socio afectivas dentro de la familia, moderando el uso de las tecnologías para que no sean éstas quienes repercutan de manera negativa hasta erradicarlas. Como parte de la investigación-acción, primero se diseñó un plan de trabajo con los alumnos que se llevó a cabo durante las dos semanas de la jornada de prácticas, el cual consistió en la sensibilización y el sentido de pertenencia dentro de un entorno familiar, resaltando la importancia, los roles familiares, el afecto, las costumbres y tradiciones, así como también el buen uso de la tecnología dentro y fuera de la escuela. Las actividades atendieron las necesidades y características de cada grupo, partiendo del grado de complejidad pertinente.

A la par de las actividades diarias en el aula, se filmaron videos que dieran evidencia de manera concreta los intereses, opiniones, sentimientos, y espontaneidades de cada uno de los alumnos ante las actividades, estas grabaciones fueron editadas y al final se les envió de manera individual a los padres de familia.

Se implementó un calendario con actividades diarias con padres de familia y/o tutores con actividades sencillas como lo es (abrazar, leer un cuento, comer juntos, jugar, platicar, etc.)

Como apoyo a las filmaciones, se les acondicionó a los alumnos un espacio en la biblioteca donde se sintieron en confianza para externar sobre su familia y los sentimientos hacia ella.

Se citó a los padres de familia a la realización de una "actividad" sin revelarles la índole de ésta, la asistencia fue mayor de la que se esperaba, por lo que se reajustaron algunas condiciones como el espacio donde se llevó a cabo.

Para iniciar con el proyecto, se presentó una plática con la Licenciada en Psicóloga Brenda Iveth Palma Mendoza acerca de este tema tan controversial, se abrió un espacio de diálogo a los padres de familia y se realizó una pequeña dinámica, a cada padre de familia se le repartió una encuesta, que tenía que ser llenada poniéndose en el papel de sus hijos, dicha encuesta había sido contestada anteriormente por sus mismos hijos en la jornada de trabajo, de igual manera elaboraron un teléfono celular de cartón, donde se metió la encuesta de cada uno. Cuando los padres terminaron de contestar, se les entregó el "teléfono celular" teniendo la frase "Tienes una 
notificación nueva", se les pidió que compararán las respuestas con la de sus hijos y vieran en cuantas habían respondido de manera correcta y que tanto era lo que conocían a sus hijos o realmente pasaban tiempo de calidad para conocer lo que cada pequeña mente pensaba, le interesaba o gustaba. Las respuestas fueron anónimas cada padre de familia se llevó consigo la reflexión de esa dinámica.

Para finalizar, se presentó un video general de cada uno de los grupos, que diera cuenta y evidencia de lo que se trabajó a lo largo del proyecto.

Al ser un proyecto de investigación cualitativa, los recursos financieros no fueron de grandes cantidades, en todo momento se procuró el economizar y/o utilizar los recursos existentes. La plática de la licenciada en psicología se costeó por parte de las maestras en formación.

\section{Resultados}

Los resultados del proyecto fueron muy satisfactorios, los instrumentos de diagnóstico arrojaron muy claro lo que de manera urgente se debe atender: que fue, el vínculo afectivo entre padres e hijos, el modo de relacionarse, la figura autoritaria y de ejemplo de los padres de familia, entre otras cosas que tienen que ver con el entorno inmediato en el que el niño está inmerso que como ya sabemos es su hogar.

La respuesta de los padres de familia, el compromiso, apoyo y trabajo hacia la atención y manejo de estas situaciones fue muy bueno, también se dio de manera favorable el ambiente durante la plática que se tuvo con ellos y que llevo a cabo la psicóloga, se atendió cada tarea y trabajo en relación al tema y la asistencia de los alumnos en el periodo de prácticas sobrepasó las anteriores.

La participación de los padres de familia el día de la actividad fue de un $85 \%$ del total, la plática fue amena y respetuosa, el espacio del diálogo fue modulado y las reacciones de las dinámicas fueron totalmente las esperadas, obteniendo buenos comentarios y reflexiones.

Por otro lado, en los alumnos se sembró a lo largo de las semanas esa gran importancia de pertenencia en el ámbito familiar, el buen uso que se le debe de dar a la tecnología dentro y fuera de la escuela, obteniendo resultados positivos en su mayoría, se implementaron escalas estimativas, listas de cotejo y rúbricas de acuerdo al grado y grupo correspondiente, midiendo los aprendizajes esperados en los alumnos acerca del tema tratado.

\section{Conclusión}

El uso de la tecnología no es necesariamente malo, sin embargo, en la actualidad se ha distorsionado la utilidad que se le debe de dar volviéndose, 
en casos muy severos, una adicción. Pero ¿Cómo es que llegamos a estos casos tan extremos sin siquiera darnos cuenta? Es un problema de raíz que afecta desde edades muy tempranas. En los niños puede tener más repercusiones de las que podemos imaginar, que pueden afectar su desarrollo físico, emocional, social y cognitivo.

Se puede decir que es un tema que toma una gran relevancia en la actualidad y que afecta a los más grandes y de igual manera a los más pequeños, la diferencia culmina en que los niños no tienen esa capacidad de conciencia que los adultos tenemos, por esta razón está en nuestras manos guiarlos y ayudarlos a aprender acerca del buen uso de la tecnología, sabemos que no es mala y que definitivamente es una gran herramienta en todo lo campos, es nuestro deber formar personas con la capacidad de manejarla y al mismo tiempo de ser conscientes con su uso y sobre todo con el tiempo que se le dedica. Viviendo en un mundo lleno de avances estamos sustituyendo personas con aparatos electrónicos, olvidando esa parte tan humana que es nuestra esencia y forma parte de nuestra vida diaria, el relacionarnos con los demás de forma directa nos beneficia en sin fin de maneras y el convertirlo en tiempos de calidad aún más.

"La tecnología no es nada. Lo importante es que tengas fe en la gente, que sean básicamente buenas e inteligentes, y si les das las herramientas, harán cosas maravillosas con ellas."

-Steve Jobs

\section{Referencias}

Barrera Valencia, C. \& Duque Gómez, L. N. (2014). Familia e internet: consideraciones sobre una relación dinámica. Revista Virtual Universidad Católica del Norte, 41, 30-44.

Bowlby, J. (1989): Una base segura. Aplicaciones clínicas de una teoría de apego. Buenos Aires: Paidós.

Henao, G., Ramírez, C. y Ramírez, L. (2007). Las prácticas educativas familiares como facilitadoras del proceso de desarrollo en el niño y niña. El Ágora, 7(2), 233-240.

Izquierdo, J. (2013). El vínculo afectivo y su importancia en la relación con tus hijos: seguridad y confianza. Siquia. 